Bull. Austral. Math. Soc.

VoL. 55 (1997) [517-519]

\title{
REGULAR GEODESIC NORMAL FORMS IN VIRTUALLY ABELIAN GROUPS
}

\author{
Walter D. Neumann and Michael Shapiro
}

We describe a virtually Abelian group $G$ generated by a finite set $X$ such that there is no regular language of geodesic $X$-words that surjects to $G$ by evaluation.

\section{INTRODUCTION}

Cannon gave an example [2, Example 4.4.2] of a virtually Abelian group with finite generating set $X$ such that the language of geodesic $X$-words for $G$ is not a regular language. Since his example admits a geodesic automatic structure in the generators $X$, it left open the possibility that this might always be so: a virtually Abelian group might admit a geodesic automatic structure for any generating set, or at least a geodesic regular unique normal form (which is weaker). This is suggested as a question in [2]. A reason to hope it might be true was that it would give a very satisfactory proof of Benson's theorem [1] that the growth function of a virtually Abelian group is rational with respect to any generating set.

In this note we exhibit a virtually Abelian group $G$ with finite generating set $X$ such that there is no regular language of geodesic $X$-words for $G$ that surjects to $G$.

In this context it is worth recalling a result of [3] that any finite generating set of an Abelian group has the property that the full language of geodesics is regular and contains an automatic structure for $G$. Moreover, for virtually Abelian groups every finite generating set can be enlarged to one with this property.

Convention. Our generating sets $X$ will always be symmetric. That is, when we list generators we mean that both those generators and their inverses are to be in our generating set.

\section{A PRELIMINARY EXAMPLE}

We start with an example that uses a weighted generating set. That is, we allow the generators to have lengths other than 1 . Our example is given by the following

Received 18th December, 1996

Both authors acknowledge support from the ARC for this research.

Copyright Clearance Centre, Inc. Serial-fee code: 0004-9729/97 \$A2.00+0.00. 
presentation:

$$
\begin{array}{r}
H=\langle x, y, t, \tau| t^{2}=\tau^{2}=[\tau, t]=1,\left(x, x^{t}, x^{\tau}, x^{t \tau}, y, y^{t}, y^{\tau}, y^{t \tau} \text { commute }\right) \\
\left.x+x^{\tau}=y+y^{t}\right\rangle .
\end{array}
$$

The last relation gives

$$
\begin{aligned}
x+x^{\tau} & =y+y^{t} \\
x+x^{\tau} & =y^{\tau}+y^{t \tau} \\
x^{t}+x^{t \tau} & =y+y^{t} \\
x^{t}+x^{t \tau} & =y^{\tau}+y^{t \tau} .
\end{aligned}
$$

These are not independent. It is not hard to see that

$$
1 \rightarrow N \rightarrow H \rightarrow V \rightarrow 1
$$

where $V$ is the Klein 4-group and $N$ is free Abelian of rank 5 . We shall give the generators $x^{ \pm 1}, y^{ \pm 1}, t^{ \pm 1}, \tau^{ \pm 1}$ lengths $1,1,1,2$ respectively. We shall suppose that there is a regular language $L$ consisting of geodesics and surjecting to $H$ and we shall derive a contradiction.

There is a homomorphism $\varepsilon: H \rightarrow \mathbb{Z}$ that takes $t$ and $\tau$ to the trivial element and $x, x^{t}, x^{\tau}, x^{t \tau}, y, y^{t}, y^{\tau}, y^{t \tau}$ all to 1 . Any element $h \in H$ thus has length at least $|\varepsilon(h)|$. Moreover, if it is in $N$ then it involves an even number of instances of $t$ and of $\tau$, so its length is congruent to $\varepsilon(h)$ modulo 2. Moreover, if it is in $N$ but not in the subgroup generated by $x$ and $y$ then it has length at least $|\varepsilon(h)|+2$, or even $|\varepsilon(h)|+4$ if it cannot be written in terms of $x, y$, and $t$.

We consider elements of $N<H$ in the positive span of $x, x^{\tau}, y$ and $y^{t}$. First consider an element of the form $x^{a} y^{b}\left(y^{t}\right)^{c}$ with $c>0$. This can be written geodesically as $x^{a} y^{b} t y^{c} t$ and thus $\ell\left(x^{a} y^{b}\left(y^{t}\right)^{c}\right)=a+b+c+2$.

We now consider an element of the form $x^{d}\left(x^{\tau}\right)^{e} y^{f}$ with $e>0$. Notice that

$$
x^{d}\left(x^{\tau}\right)^{e} y^{f}=x^{d-\delta}\left(x^{\tau}\right)^{e-\delta} y^{f+\delta}\left(y^{t}\right)^{\delta}
$$

Thus, if $e \leqslant d$, this lies in the positive span of $x, y$, and $y^{t}$ and thus $\ell\left(x^{d}\left(x^{\tau}\right)^{e} y^{f}\right)=$ $d+e+f+2$. On the other hand, if $e>d$, then it is not hard to see that $x^{d}\left(x^{\tau}\right)^{e} y^{f}$ has length $d+e+f+4$. And moreover, if $e>d+1$ then every geodesic for this element has the form

$$
w=w_{1} \tau x^{e} \tau w_{2}
$$


where $w_{1}$ and $w_{2}$ are positive words in $x$ and $y$ which together contain $d x$ 's and $f y$ 's. (If $e=d+1$ we have additional geodesics such as $x^{-1} y^{f+e} t y^{e} t$ of length $2 e+f+3=d+e+f+4$.) Notice that the word $(*)$ is geodesic if and only if $e>d$. Now $L$ must contain such words with $d$ arbitrarily large. We fix a finite state automaton $M$ for $L$ and choose a word $w$ so that $d$ is at least the number of states of $M$. Then $e$ is greater than the number of states of $M$ so the portion $x^{e}$ of $w$ traverses a loop of $M$. By eliminating such loops we find a word $w^{\prime}=w_{1} \tau x^{e^{\prime}} \tau w_{2}$ which is also in $L$, but has $e^{\prime}$ no larger than the number of states of $M$ and hence no larger than $d$. It is thus not geodesic. Thus there can be no regular language of geodesics surjecting to $H$.

\section{The MAin EXAMPLe}

We now embed the above preliminary example in an example where all generators have length 1 . We take

$$
\begin{aligned}
G=\langle x, y, t, s| t^{2}=s^{4}=[s, t] & =1,\left(x^{\alpha}, x^{\beta}, y^{\alpha}, y^{\beta}\right. \text { commute, for any } \\
& \left.\left.\alpha, \beta \in\left\{1, s, s^{2}, s^{3}, t, t s, t s^{2}, t s^{3}\right\}\right), x+x^{s^{2}}=y+y^{t}\right\rangle .
\end{aligned}
$$

$G$ has a finite index normal subgroup which is free Abelian of rank 10. Taking $\tau=s^{2}$ embeds $H$ in to $G$. It is easy to see that this embedding is totally geodesic in the sense that a geodesic word in $x, y, s, t$ that evaluates into the subgroup is the result of substituting $\tau=s^{2}$ in a geodesic word of the subgroup. We leave this to the reader. It then follows that this example inherits the property that the language of geodesics has no regular sublanguage which surjects to the group (in fact, no regular sublanguage can evaluate to a subset containing the subgroup).

\section{REFERENCES}

[1] M. Benson, 'Growth series of finite extensions of $\mathbb{Z}^{n}$ are rational', Invent. Math. 73 (1983), 251-269.

[2] D.B.A. Epstein, J.W. Cannon, D.F. Holt, S.V.F. Levy, M.S. Paterson and W.P. Thurston, Word processing in groups (Jones and Bartlett, 1992).

[3] W.D. Neumann and M. Shapiro, 'Automatic structures, rational growth and geometrically finite groups', Invent. Math. 120 (1995), 259-287.

Department of Mathematics

The University of Melbourne

Parkville Vic 3052

Australia

e-mail: neumann@maths.mu.oz.au

shapiro@maths.mu.oz.au 